We propose a formal model to explain the mutual influence between observed behavior and subjects' elicited beliefs in an experimental sequential prisoner's dilemma. Three channels of interaction can be identified in the data set and we argue that two of these effects have a non-classical nature as shown, for example, by a violation of the sure thing principle. Our model explains the three effects by assuming preferences and beliefs in the game to be complementary. We employ non-orthogonal subspaces of beliefs in line with the literature on positive-operator valued measure. Statistical fit of the model reveals successful predictions.

Keywords: positive-operator valued measure, preferences, beliefs, consensus effect, sequential prisoner's dilemma

\title{
A quantum-like model for complementarity of preferences and beliefs in dilemma games
}

\author{
Jacob Denolf ${ }^{\mathrm{a}, 1}$, Ismael Martínez-Martínez ${ }^{\mathrm{b}, 2}$, Haeike Josephy, ${ }^{\mathrm{a}, 3}$, Albert \\ Barque-Duran ${ }^{\mathrm{c}, 4}$ \\ ${ }^{a}$ Department of Data Analysis, Ghent University, H. Dunantlaan 1, 9000 Ghent, Belgium \\ ${ }^{b}$ Düsseldorf Institute for Competition Economics (DICE), Heinrich Heine Universität \\ Düsseldorf, Universitätsstraße 1, D-40225 Düsseldorf, Germany \\ ${ }^{c}$ Department of Psychology, City University London, London, ECIV OHB, UK
}

\footnotetext{
${ }^{1}$ Corresponding author. Email address: jacob.denolf@ugent.be

${ }^{2}$ Email address: ismael@imartinez .eu

${ }^{3}$ Email address: haeike. josephy@ugent. be

${ }^{4}$ Email address: albert.barque-duran@city.ac.uk
} 


\section{Introduction}

During the recent decade, there is an increasing interest in decision-making and cognitive models that employ a quantum probabilistic $(Q P)$ framework. In fact, the application of quantum-like concepts to portray human information processing was considered since the early development of quantum mechanics. For example, Bohr (1950) defended the idea that some aspects of quantum theory could provide an understanding of cognitive processes but never provided a formal cognitive model in light of a QP hypothesis. The so called quantum cognitive theories have only begun to emerge as of late (Busemeyer and Bruza, 2012; Deutsch, 1999; Haven and Khrennikov, 2013; Khrennikov, 2010; Pothos and Busemeyer, 2013; Wang et al., 2014; Yearsley and Pothos, 2014).

QP is defined as the set of mathematical rules used to assign probabilities to events from quantum mechanics (Hughes, 1989; Isham, 1989), but without any of the physics. As it is derived from a different sets of axioms than classical probability theory, it is subject to alternative constraints and has the potential to be relevant in any area of science where a need to formalize uncertainty arises. Since encoding uncertainty is a major aspect of cognitive functions in psychology, QP shows potential for cognitive modeling. These studies are not about the use of quantum physics in brain physiology, which is a disputable issue (Hameroff, 2007; Litt et al., 2006) about which we are skeptical. Rather, we are interested in QP theory as a mathematical framework for cognitive modeling.

Applications of QP theory have been presented in decision-making (Bordley, 1998; Busemeyer et al., 2011, 2006; Lambert-Mogiliansky et al., 2009; Pothos and Busemeyer, 2009; Trueblood and Busemeyer, 2011; White et al., 2014; Yukalov and Sornette, 2011), conceptual combination (Aerts, 2009; Aerts and 
Gabora, 2005; Blutner, 2008), memory (Bruza, 2010; Bruza et al., 2009), and perception (Atmanspacher et al., 2004). For a detailed study on the potential use of quantum modeling in cognition, see Busemeyer and Bruza (2012) and Pothos and Busemeyer (2013). The majority of models presented in the quantum cognition literature addresses standard aspects of decision-making processes: similarity judgments (Barque-Duran et al., 2016; Pothos et al., 2015; Yearsley et al., 2014), the constructive role of articulating impressions (White et al., 2015, 2014), and order effects in belief updating (Trueblood and Busemeyer, 2011) among numerous other applications.

Little literature has focused on strategic decision-making or game theory. Whenever two or more agents interact, one agent is not only reacting to the information that he receives, but is likewise generating information towards other players. These strategic environments are unique in relation to standard decision-making scenarios under uncertainty, since every agent needs to reason on two parts of the problem: his own actions and his expectations on the opponent's actions. Few studies applying QP instruments to model the way agents process the information in a game have been published with regards to this particular matter: Pothos and Busemeyer (2009), Pothos et al. (2011), Busemeyer and Pothos (2012), and Martínez-Martínez and Sánchez-Burillo (2016). Other approaches in which the quantumness enters through an extension of the classical space of strategies and/or signals have also been discussed, e.g., by La Mura (2005), Brandenburger (2005), and Brunner and Linden (2013); as well as a model to analyze games with agents exhibiting contextual preferences (LambertMogiliansky and Martínez-Martínez, 2015).

In this paper, we describe the application of QP theory to modeling the 
mutual influence between preferences and beliefs in sequential social dilemmas. This idea was first explored in Martínez-Martínez et al. (2015). We present a quantum-like model for preferences and beliefs $(\mathrm{QP} \& \mathrm{~B})$ that replicates the experimental results from Blanco et al. (2014) while providing a novel theoretical approach on cognitive dynamics in strategic interactions. Our model asserts that the relationship between a player's beliefs and his preferences is inherently nonclassical and continues the work done in Pothos and Busemeyer (2009) exploiting the ideas of measurement utilized in quantum theory. We redefine these two properties as complementary. In that capacity, they cannot be measured at the same time, as the act of measuring one property alters the state of the other property. The non-classical nature of such a relationship and its application in cognition has already been discussed in, e.g., Denolf and Lambert-Mogiliansky (2016).

\section{Experimental design}

The data set that our QP\&B model deals with is provided by Blanco et al. (2014). Their experiment was designed for explicitly testing different channels through which preferences and beliefs of an agent immersed in a social dilemma may influence each other. As the authors motivate, this experimental evidence is novel and its main interest stems from the fact that previous analyses of strategic interactions considered preferences and beliefs to be independent. This fact implies that the choice of actions in environments with uncertainty can be rationalized as just a best-response to some particular form of belief about the possible states of the world or about the action that is expected to be played by an opponent. 


\subsection{Standard version of the prisoner's dilemma game}

The symmetric prisoner's dilemma game is a game involving two players, player $I$ and player $I I$, who can choose among two actions: cooperate $(C)$ or defect $(D)$. The normal form of this game is defined by the following $2 \times 2$ payoff matrix

\begin{tabular}{|c|c|c|c|}
\hline & \multicolumn{2}{|c|}{ Player II } \\
\hline & & $C$ & $D$ \\
\hline & $C$ & $\left(\pi_{c}, \pi_{c}\right)$ & $\left(\pi_{b}, \pi_{a}\right)$ \\
\hline & $D$ & $\left(\pi_{a}, \pi_{b}\right)$ & $\left(\pi_{d}, \pi_{d}\right)$ \\
\hline
\end{tabular}

where the payoff entries satisfy the inequalities $\pi_{a}>\pi_{c}>\pi_{d}>\pi_{b}$.

The scheme of possible results of payoffs is as follows. If player $I$ decides to cooperate, $I$ can receive the second best possible outcome $\pi_{c}$ if the opponent $I I$ also cooperates, but I's attempt to cooperate is exposed to being exploited by II if $I I$ decides to defect. In the latter scenario, $I I$ would collect the best outcome of value $\pi_{a}$ while leaving $I$ with the lowest payoff $\pi_{b}$. If player $I$ decides to defect, then this player is guaranteed not to obtain the lowest payoff, but at least an amount $\pi_{d}$ if player $I I$ defects as well. If player $I I$ decided to cooperate, then $I$ is taking advantage of the situation and obtaining the maximum benefit $\pi_{a}$.

Technically, we say that mutual defection is the Nash equilibrium of this game because there is no unilateral deviation that could make the deviating player earn more, while mutual cooperation is the Pareto optimal situation. Therefore, this game represents a social dilemma for the players: the individual choice of defection dominates the attempt to cooperate for any given choice of the opponent, which is not socially optimal. Why is this a dilemma? Because this game formalizes a conflict between the individual (the Nash equilibrium) and the collective (Pareto optimal) level of reasoning: if both players actually choose to 
(a)

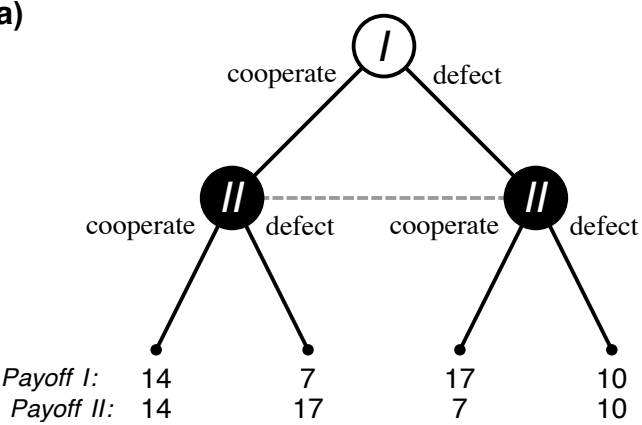

(b)

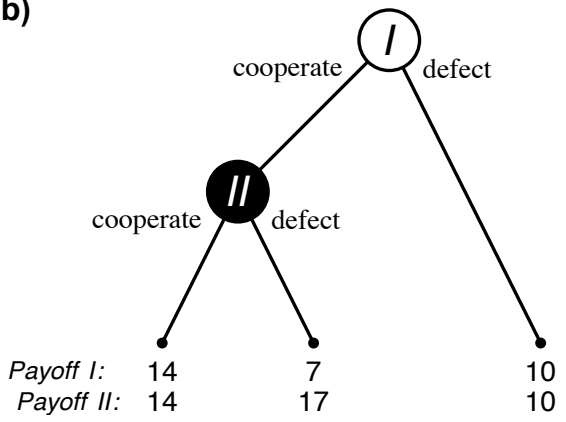

Figure 1: (a) Standard (simultaneous) Prisoner's Dilemma. (b) Sequential Prisoner's Dilemma.

defect, both of them generate a total payoff of $2 \times \pi_{d}$, which is by definition lower than the aggregate payoff if both of them coordinated in full cooperation, $2 \times \pi_{c}$.

The standard version of the prisoner's dilemma game is a one-shot strategic interaction with simultaneous moves by the opponents. This implies that both players make their own individual decision (whether to cooperate or not) without knowing what the opponent is choosing. Once both players have chosen their strategy, both actions become public and the payoffs are generated.

Each player reacts to his own belief or expectation on the opponent's intention, and as a consequence, the preferred action in the dilemma crucially depends on the way players form their beliefs about the opponent moves. Therefore, it is important to understand how beliefs and preferences do (or do not) influence each other in this decision-making process. ${ }^{5}$

\subsection{Sequential prisoner's dilemma}

The experiment conducted by Blanco et al. (2014) focuses on a variation of the Prisoner's Dilemma game discussed above: a sequential one. In Fig. 1 we

\footnotetext{
${ }^{5}$ See Blanco et al. (2014, Section 1) about possible correlations between preferences and beliefs in dilemmas with models of social preferences such as inequality aversion and reciprocal preferences.
} 


\footnotetext{
${ }^{6}$ In the belief-elicitation task, the players were asked how many of the other participants (potential rivals for the play of the game) cooperate in the role of Player II. This task is incentivized
} 


\begin{tabular}{r|lll}
\hline Treatment & Baseline & Elicit_Beliefs & True_Distribution \\
\hline \hline Task 1 & 2nd move $(I I)$ & 2nd move $(I I)$ & 2nd move $(I I)$ \\
Feedback on $I I$ & No & No & Yes \\
Task 2 & 1st move $(I)$ & beliefs (about $I I)$ & 1st move $(I)$ \\
Task 3 & beliefs (about $I)$ & 1st move $(I)$ & beliefs (about $I)$ \\
\hline \# Participants & 40 & 60 & 60 \\
\hline
\end{tabular}

Table 1: Experimental treatments in Blanco et al. (2014, Table 1).

each task is performed and this allows to measure different correlations between actions (which are supposed to proxy the preferences of the players) and beliefs. We now briefly explain the three different treatments, which are also summarized in Table 1.

\subsection{Experimental treatments}

Ten subjects participate in each session. For each of the following treatments, several sessions were conducted. The total numbers of participants are displayed in Table 1.

Baseline. This treatment can be considered as a mere control group, such that the subjects play the game in its natural structure, with no attention paid to observing their beliefs. The players first choose what their action II will be and no information is revealed to them so that the participants' beliefs are not exogenously influenced. Subsequently, they choose what their action for the role of $I$ will be, and finally they are given a meaningless question about their beliefs on the global rate of cooperation in the group of first movers. The informational gain of this last task is void because its only use is to balance the different treatments making their length comparable (both in time and the number of tasks).

with a quadratic scoring rule rewarding the accuracy of the stated beliefs: players earn more the closer their prediction is to the actual rivals' cooperation rate (Blanco et al., 2014, Equation 3). 


\begin{tabular}{r|llll}
\hline Treatment & Baseline & Elicit_Beliefs & True_Distribution & Total \\
\hline \hline First mover (Player I) & $27.5 \%$ & $55.0 \%$ & $56.7 \%$ & $48.8 \%$ \\
Second mover (Player II) & $55.0 \%$ & $53.3 \%$ & $55.0 \%$ & $54.4 \%$ \\
\hline
\end{tabular}

Table 2: Average cooperation rates by treatment in the experiment by Blanco et al. (2014), also labeled as Table 2 in their original paper.

Elicit_Beliefs. In this treatment, the players first choose what their action II will be, and then they have to reveal their belief about the rate of cooperation that they will receive from the second movers. Finally, they have to choose their action $I$. Thus, this treatment introduces a belief-measurement between the two choices of actions. This allows us to explore the effect of a measurement of the beliefs about the move by opponent II on the choice of action $I$.

True Distribution. This treatment presents a somewhat 'similar' sequence of tasks for the players compared to the previous treatment Elicit_Beliefs. The players begin by choosing their action II. Then, they are told what the true cooperation rate for action $I I$ was in their group. They finish by choosing the action $I$. This treatment differs from the previous one in that this time, the forecast of the opponents' move is not a belief generated by the players themselves, but true information being released to them exogenously.

\section{Aggregate behavior and basic modeling}

Table 2 presents the aggregate results of the three experimental treatments. First off, we cannot observe any significant difference in the cooperation rates as a second mover between treatments. This is to be expected as the question (measurement) regarding the choice of action in the role of player II is identical 


\footnotetext{
${ }^{7}$ Note especially that it is the first measurement performed in all treatments and therefore, it is not subject to the effects targeted by this experimental design.
} 
incentivized elicitation of beliefs impacts the state of the subjects participating in the experiment similarly to an update of beliefs via the acquisition of true information revealed exogenously.

\subsection{Violation of the sure thing principle}

The differences in first move cooperation rates reveal the presence of a violation of the sure thing principle in the data, as

$$
27.5 \%=p\left(C_{I}\right) \neq \sum_{i} p\left(C_{I} \mid B_{i}\right)=55 \%
$$

with $C_{I}$ the event of the player cooperating on the first move and $B_{i}$ the event of the player answering that he thinks $i$ opponents cooperate during the belief elicitation. This in turn points out the interest in using a quantum-like model to describe the behavior of the participants in this experiment, since classical statistics cannot account for them in a simple manner, while quantum-like easily do.

\subsection{The simplest quantum-like model}

In the remaining of Section 3, we illustrate the basic mechanics of quantumlike toy models designed to address the issue of measurement as well as construct different building blocks that will be fully developed later. As the reader will see, Section 4 integrates them in a unified model. Now, we only show which aspects of quantum-like modeling can account for the empirical effects observed in the data set, without taking into account how they correlate to form the proper model.

We introduce the most basic quantum-like model to represent concepts such as actions, preferences and beliefs in quantum-like terms (observables, measurements and orthonormal basis of their outcomes) and use projective measurements (with their resulting probabilities) to explain the first results observed in the data from Blanco et al. (2014). We consider the preferences 
of an agent as the individual's attitude toward the different elements of a set of outcomes, to be reflected in the choices observed along the sequence of decisions (Lichtenstein and Slovic, 2006). In this case, and because of the strategic nature of this decision-making process, the outcomes (possible payoffs to be obtained) depend on the actions (cooperate or defect) a players chooses, but also on the choices made by a rival.

The actions of a player can be represented by two orthogonal vectors $|C\rangle$ (for cooperation) and $|D\rangle$ (for defection). The two vectors form an orthonormal basis and span a bi-dimensional Hilbert space $\mathcal{H}_{i}$ with $i \in\{I, I I\}$ denoting the role in the game as player $I$ or $I I$ for which such action is chosen. ${ }^{8}$ The player is considered to be in a superposition over these actions, being represented by a normalized state vector $|S\rangle$. The projection of the state vector onto the elements of the orthonormal basis defines the probability that the player chooses each of the actions, as a proxy of her preferences.

We consider the beliefs as the distribution with which the agents judge the likelihood of realization of each possible relevant state of the world. The possible states in this setting concern the possible cooperation of opponents, as this, together with one's own actions, determines the outcome of the game.

\footnotetext{
${ }^{8}$ For the finite dimensional case, a Hilbert space $\mathcal{H}$ is a linear space endowed with a scalar product $\left\langle\psi_{1} \mid \psi_{2}\right\rangle \in \mathbb{R}$. Its elements (or states) are denoted by $|\psi\rangle \in \mathcal{H}$. If the state of the system is $|\psi\rangle$ we say it is in a pure state. The projector $P_{\psi}=|\psi\rangle\langle\psi|$, an operator acting on $\mathcal{H}$ as $P_{\psi}|\phi\rangle=\langle\psi \mid \phi\rangle|\psi\rangle$, has a bijective relation with $|\psi\rangle$, and we can describe the state $|\psi\rangle$ in terms of $P_{\psi}$. Any element or vector of the space of states is called a ket-vector and represented by $|\cdot\rangle$, and we have the dual space of the $b r a$-vectors, symbolized by $\langle\cdot|$. Hilbert spaces are generally defined over the field of complex numbers, but in this paper it is enough to work only with reals. Note that given a state $|\psi\rangle$ associated to a vector $\psi \in \mathbb{R}^{N}$, we obtain $\langle\psi|$ associated to $\psi^{T}$, where ${ }^{T}$ is the operation of vector transposition. The name of bra-ket (or Dirac's) notation comes from splitting the bracket $\langle\cdot \cdot \cdot\rangle$ representing the scalar product, which is the crucial operation to compute probabilities in this framework.
} 


\footnotetext{
${ }^{9}$ The probability of observing an outcome is calculated as the square of the norm of the projection of the state vector onto the subspace spanned by the vectors representing the outcome. When the outcome is represented by only one vector (simplest case), this calculation reduces to the square of the inner product of the state vector and the outcome vector. The act of measurement changes the state vector of the system from an initial state to a post-measurement state, by projecting (and normalizing) the state vector onto the subspace spanned by the outcome vectors. Projective measurements deal naturally with incompatible measurements, and note also that when they are performed on a density matrix diagonal in a particular basis, they are equivalent to Bayesian updates.
} 
probability of the player defecting as second mover is:

$$
p\left(D_{I I}\right)=\| P_{D_{I I}}\left|S_{I I}\right\rangle \|^{2}=\left\langle D_{I I} \mid S_{I I}\right\rangle^{2}=d_{I I}^{2},
$$

with $P_{D_{I I}}=\left|D_{I I}\right\rangle\left\langle D_{I I}\right|=\operatorname{diag}(0,1)$ the projector on $\left|D_{I I}\right\rangle$. This outcome would likewise project the state vector onto its post-measurement state $\left|S_{I I}^{\prime}\right\rangle=\left|D_{I I}\right\rangle$. The normalization restriction on the state vector implies that total probabilities add up to one, $c_{I I}^{2}+d_{I I}^{2}=1$. From the cooperation rates as player II reported in Table 2, we can estimate these through our sample as:

$$
\hat{c}_{I I}^{2}=0.544 \text { and } \hat{d}_{I I}^{2}=0.456 \text {. }
$$

Note that we estimate by taking the average cooperation rates across the treatments, because we have justified above that they are not significantly different from one another.

We can model the choice of the players for their action as player $I$ in the Baseline condition in a Hilbert space $\mathcal{H}_{I} \equiv \mathbb{R}^{2}$, with the basis $\left\{\left|C_{I}\right\rangle,\left|D_{I}\right\rangle\right\}$. The state vector is now

$$
\left|S_{I}\right\rangle=c_{I}\left|C_{I}\right\rangle+d_{I}\left|D_{I}\right\rangle
$$

and we can infer from the data (Table 2, column 1) that

$$
\hat{c}_{I}^{2}=0.275 \text {, and } \hat{d}_{I}^{2}=0.725 \text {. }
$$

In this case, we only consider the cooperation and defection rates in the Baseline treatment. Because of the significant difference in the cooperation rate as player $I$ across treatments, considering the average is not sensible (see discussion in Section 3). 


\begin{tabular}{l|rrrrrrrrrr}
\hline \#Cooperators (Belief) & 0 & 1 & 2 & 3 & 4 & 5 & 6 & 7 & 8 & 9 \\
\hline \hline Abs. frequency (out of 60 subjects) & 5 & 2 & 5 & 5 & 12 & 9 & 9 & 6 & 4 & 3 \\
\hline
\end{tabular}

Table 3: Number of players in treatment Elicit_Beliefs expecting each possible number of cooperators in their session.

Finally, we model the beliefs of the players in the Hilbert space $\mathcal{H}_{B}$, (spanned by $\left.\left\{\left|B_{j}\right\rangle\right\}\right)$. The normalized state vector is

$$
\left|S_{B}\right\rangle=\sum_{j=0}^{9} b_{j}\left|B_{j}\right\rangle .
$$

From the data regarding the Elicit_Beliefs treatment (see Table 3), we get that

$$
\begin{gathered}
\hat{b}_{0}^{2}=5 / 60, \hat{b}_{1}^{2}=2 / 60, \hat{b}_{2}^{2}=5 / 60, \hat{b}_{3}^{2}=5 / 60, \hat{b}_{4}^{2}=12 / 60, \\
\hat{b}_{5}^{2}=9 / 60, \hat{b}_{6}^{2}=9 / 60, \hat{b}_{7}^{2}=6 / 60, \hat{b}_{8}^{2}=4 / 60, \hat{b}_{9}^{2}=3 / 60 .
\end{gathered}
$$

\section{Building blocks}

\subsection{Three effects}

Effect 1 (Consensus effect). Proof of and an extensive discussion on the presence of this effect is presented in Blanco et al. (2014) where it is shown that players' beliefs are biased towards their own actions. As such, a player who cooperates as second mover will expect a higher second-mover cooperation rate amongst the other players. A visualization of this effect can be found in Fig. 2. Viewing this in light of the performed measurements, the consensus effect denotes the influence of second mover action measurements on the beliefs of the same participant.

Effect 2 (Reasoned player). The second effect is the influence that belief measurements have on action measurements. As these actions are driven by one's 


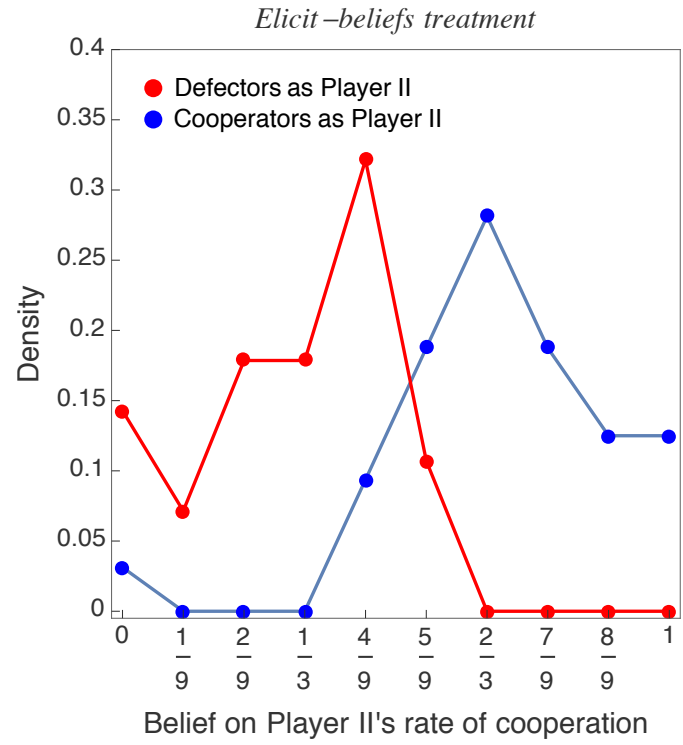

Figure 2: Second move defecting players (red line) believe that less opponents will cooperate. Second move cooperating players (blue line) believe more opponents will cooperate. The second move action was measured before the beliefs.

preferences, this effect encompasses the influence of the belief measurements on the preferences of the same player. We claim that the act of eliciting the beliefs of the player fundamentally changes this player even when disregarding the exact outcome of this belief measurement. When the player is asked to form an opinion about the cooperation rate of his opponents, this changes him into a more reasoned state about the opponent, in opposition to a more intuitive state when not explicitly asked to form this opinion. In the data, this can be viewed in the violation of the sure thing principle discussed in Section 3.1. The average first move cooperation rate of players, after forming explicitly their beliefs about the cooperation of the opponent (Elicit_Beliefs), is twice as large as the average first move cooperation rate of players, in which beliefs were not elicited (Baseline) (see Table 2). Nevertheless, this cooperation rate in the Elicit_Beliefs group is not 
differing significantly from the cooperation rate in the True Distribution group. In this group, participants received full information about the cooperation rate of the opponents and are therefore assumed to make a more deliberate decision. Since these cooperation rates are similar, we can assume that players are in a similar reasoned state in the Elicit_Beliefs group.

Effect 3 (Classical correlation). The third effect we discuss is the correlation between a player's first and second move. This is observed in all three conditions, as noted in Results 1, 2 and 3 from Blanco et al. (2014). That is, first move cooperators are likely to also cooperate on the second move and vice versa. We concur with Blanco et al. that this correlation is exhibited mostly through an indirect belief-based channel. This way, we attempt to include the observed correlation as a logical consequence of our previously described effects. The second move action measurement influences the first move action measurement through a player's beliefs. We can assume this correlation to be classical in nature, as opposed to the two other effects.

\subsection{Compatible and incompatible measurements}

Roughly speaking, two measurements $M_{1}$ and $M_{2}$ are considered incompatible if the order in which the measurements are done changes the outcome, as the act of performing one measurement influences the other measurements regardless of the outcome. Mathematically speaking, this means that one or more projector matrices associated with outcomes of measurement $M_{1}$ do not commute with one or more projector matrices associated with outcomes of measurement $M_{2}$. If two measurements are maximally incompatible, no projector matrix associated with an outcome of measurement $M_{1}$ commutes with a projector matrix associated with an 
outcome of measurement $M_{2}$, and they are called complementary. As such, both measurements $M_{1}$ and $M_{2}$ cannot be performed together, as the act of performing one of the measurements (without specifying its outcome), influences the other measurement. These concepts elegantly deal with situations where violations of the sure thing principle emerge.

We will consider the belief elicitation to be complementary with the action measurements, as this explains both the consensus effect and the reasoned player effect. This approach should not come as a surprise. First, using complementarity as an explanation for the consensus effect is argued in Busemeyer and Pothos (2012) where the consensus effect is seen as a form of social projection. Second, the idea of the player being more reasoned can be seen as a violation of the sure thing principle. These violations are a prime indicator of measurements not commuting which is the definition of incompatible measurements. We will now show how the projective measurement formalism deals with our hypothetically compatible (first and second move actions) and incompatible (actions and beliefs) measurements.

When two measurements are considered compatible, the Hilbert spaces representing the outcomes of these measurements can be tensored to construct a larger Hilbert space spanned by vectors that now represent joint outcomes. As argued before, we assume the first move action and second move action to be compatible, as they are considered to be measurable at the same time. Therefore, the Hilbert space which models the relationship between both is $\mathcal{H}_{I} \otimes \mathcal{H}_{I I}$, spanned by $\{|C C\rangle,|C D\rangle,|D C\rangle,|D D\rangle\}$, with $|C D\rangle=\left|C_{I}\right\rangle \otimes\left|D_{I I}\right\rangle$ (other vectors defined similarly). The player is represented by a normalized state vector:

$$
|S\rangle=s_{C C}|C C\rangle+s_{C D}|C D\rangle+s_{D C}|D C\rangle+s_{D D}|D D\rangle .
$$


360

We now provide two examples of how probabilities are calculated within this Hilbert space. The other relevant probabilities are calculated in a similar way. The projector and probability associated with a player defecting on the role of $I$, but cooperating on the role of $I I$ is

$$
P_{D C}=P_{D_{I}} \otimes P_{C_{I I}}=\left(\begin{array}{ll}
0 & 0 \\
0 & 1
\end{array}\right) \otimes\left(\begin{array}{ll}
1 & 0 \\
0 & 0
\end{array}\right)=\left(\begin{array}{llll}
0 & 0 & 0 & 0 \\
0 & 0 & 0 & 0 \\
0 & 0 & 1 & 0 \\
0 & 0 & 0 & 0
\end{array}\right),
$$

so

$$
p(D C)=\| P_{D C}|S\rangle \|^{2}=s_{D C}^{2} .
$$

The projector and probability associated with the player cooperating on the second move (without specifying a choice as player $I$ ), are:

$$
P_{. C}=I^{2} \otimes P_{C_{I I}}=\left(\begin{array}{ll}
1 & 0 \\
0 & 1
\end{array}\right) \otimes\left(\begin{array}{ll}
1 & 0 \\
0 & 0
\end{array}\right)=\left(\begin{array}{llll}
1 & 0 & 0 & 0 \\
0 & 0 & 0 & 0 \\
0 & 0 & 1 & 0 \\
0 & 0 & 0 & 0
\end{array}\right),
$$

and

$$
p(. C)=\| P_{. C}|S\rangle \|^{2}=s_{C C}^{2}+s_{D C}^{2} .
$$

Directly from the data (for the Baseline treatment), we derive

$$
\hat{s}_{C C}^{2}=0.25, \hat{s}_{C D}^{2}=0.025, \hat{s}_{D C}^{2}=0.3, \text { and } \hat{s}_{D D}^{2}=0.425 \text {. }
$$

This models the (classical) correlation between first and second move, as noted above in Effect 3. 
Incompatible measurements are represented by different bases in the same Hilbert space (as opposed to one tensored basis for compatible measurements). To model the relationship between the choice of action in the role of player $I$ and the beliefs that a player holds, we could use a Hilbert space $\mathcal{H}_{I, B}$ of large enough dimensionality to present 10 orthogonal subspaces, each one representing one belief. As such, we would need at least a 10-dimensional space, with 10 orthonormal vectors forming the belief basis. In such 10-dimensional Hilbert space, the 2 possible outcomes of the first movement action are each represented by orthogonal 5-dimensional subspaces.

The Hilbert space $\mathcal{H}_{I I, B}$, which models the relationship between the belief measurement and the second movement action would be similarly spanned by 10 orthonormal basisvectors, each one representing an outcome of the belief measurement. The outcomes of the second movement action are also represented by 5 -dimensional subspaces. The rules for projection and calculating probabilities remain the same. The probability of an outcome of a measurement is still the square of the norm of the projection of the state vector on the relevant subspace. The act of measuring still changes the superposition of the state vector, projecting and normalizing it onto the relevant subspace.

In summary, the relationship between the belief and action measurement is represented by the description of the action subspaces in terms of the belief basis. In such setting, the consensus effect would be represented by the form of the 5dimensional action subspaces in $\mathcal{H}_{I I, B}$, while the effect of the player becoming more reasoned would be represented by the form of the 5-dimensional action subspaces in $\mathcal{H}_{I, B}$. 


\subsection{A very basic model}

We can attempt to construct a model which successfully incorporates all three effects, by combining how we modeled the compatible action measurements, with how we could model the incompatible belief and action measurements. The standard procedure from quantum-like measurement theory tells us to construct the Hilbert space $\mathcal{H}^{\text {orth }}=\mathcal{H}_{I, B} \otimes \mathcal{H}_{I I, B}$. This is a 100-dimensional Hilbert space, with 2 orthogonal 50-dimensional subspaces representing the actions in role $I, 2$ orthogonal 50-dimensional subspaces representing the actions in role $I I$, and 10 orthogonal 10-dimensional subspaces representing the possible beliefs. As the first and second move actions are considered compatible, they can be measured at the same time. As such, the 4 possible joint outcomes of the action measurements are represented by four 25-dimensional subspaces.

The player would be represented by a normalized state vector in this 100-dimensional Hilbert space, from which the relevant probabilities can be calculated. From a statistical point of view this state vector already provides us with 99 degrees of freedom (we lose 1 as the state vector is normalized), without even delving into how many degrees of freedom pop up due to the different 10-, 25- and 50-dimensional subspaces used in this construction. As we have 160 data points, this elementary model would be by no means elegant, and a statistical fit is not feasible because of being greatly overparametrized. One solution is to impose further restrictions on the state vector and/or on the different outcome subspaces, for example, by allowing only state vectors within a certain subspace or assuming a certain distribution over the resulting probabilities. The form of these restrictions is, however, an open question at this point.

In the following section we show how a small deviation from the most 
common quantum-like approach allows us to reduce the complexity of the total Hilbert space to only four dimensions. We use a less structured set of planes to represent beliefs which provides a truly intuitive connection between the different elements of the model.

\section{The model for Quantum-like Preferences and Beliefs}

\subsection{A new belief basis}

To diminish the problematic dimensionality of $\mathcal{H}_{B}$ we let the vectors $\left|B_{i}\right\rangle$ (the outcomes of the belief elicitation) be non-orthogonal because otherwise, the 10 orthogonal vectors would span a 10-dimensional Hilbert space. Next to making the dimension of $\mathcal{H}_{B}$ sufficiently small, this modification will allow us to model some implicit structure between the different outcomes and will link the construction of these beliefs directly to the approach of Pothos and Busemeyer (2009) to the standard prisoner dilemma. Roughly speaking, in Pothos and Busemeyer (2009), the emergence and evolution of the player's beliefs about his opponent's behavior is represented by a rotation of the state vector in the Hilbert space. While in Pothos and Busemeyer (2009) this rotation is defined by a Hamiltonian with a parameter $\gamma$, we now have the means to explicitly incorporate the elicited beliefs into our model. To do so, we redefine the belief-vectors $\left|B_{i}\right\rangle$ in a 2-dimensional Hilbert space, with $\left|B_{0}\right\rangle$ and $\left|B_{9}\right\rangle$ orthogonal and the other $\left|B_{i}\right\rangle$ in between them. For simplicity, we will assume the distribution of the $\left|B_{i}\right\rangle$ ( $i \neq 0,9)$, to be uniform between $\left|B_{0}\right\rangle$ and $\left|B_{9}\right\rangle$, yielding an angle $\pi /(2 \times 9)$ between all $\left|B_{i}\right\rangle$ and $\left|B_{i+1}\right\rangle$. This provides us with an elegant, parameter free (as the '9' is endogenous to the game) form of the vectors representing the outcomes of the belief elicitation. This is a simple first approach to the exact distribution of the $\left\{\left|B_{i}\right\rangle\right\}_{i=0}^{9}$, which can be adjusted or made more complex if necessary. This 


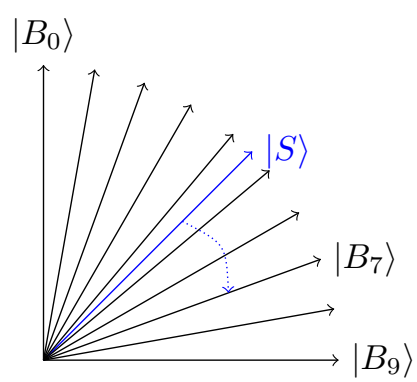

Figure 3: The redefined $\left|B_{i}\right\rangle$. A player thinking 7 out of 9 opponents cooperate projects the state vector onto $\left|B_{7}\right\rangle$.

effectively makes the players development of her explicit beliefs to be represented by a rotation of the state vector, as in Pothos and Busemeyer (2009). Our view differs from Pothos and Busemeyer (2009) in the sense that we still want to make predictions and derive probabilities from this rotation, using the standard rules of projective measurements: an outcome and its probability as defined by a projector on the relevant subspace. This approach also models an implicit order between the different outcome vectors (e.g. $\left|B_{i}\right\rangle$ being 'in between' $\left|B_{i-1}\right\rangle$ and $\left.\left|B_{i+1}\right\rangle\right)$, something lacking in the previous approach where all belief vectors were orthogonal. This idea is depicted in figure 3.

With the redefined 2-dimensional Hilbert Space $\mathcal{H}_{B}$, we rebuild the Hilbert space $\mathcal{H}_{Q P \& B}$ which contains the representations of all measurements, as well as their correlations. As we redefined our vectors $\left|B_{i}\right\rangle$ representing the elicited beliefs, the projectors onto these vectors will also have a new form:

$$
\left|B_{i}\right\rangle\left\langle B_{i}\right|=\left(\begin{array}{cc}
\cos ^{2}\left(\frac{i \pi}{18}\right) & \cos \left(\frac{i \pi}{18}\right) \sin \left(\frac{i \pi}{18}\right) \\
\cos \left(\frac{i \pi}{18}\right) \sin \left(\frac{i \pi}{18}\right) & \sin ^{2}\left(\frac{i \pi}{18}\right)
\end{array}\right),
$$

with $i=0,1, \ldots 9$.

To incorporate a measurement with non-orthogonal outcome vectors, we will go beyond the basic procedure of quantum measurement as done in Section 3.2. 
To do so, we present two options, one favoring quantum theoretic consistency and one favoring a simpler experimental interpretation. Note that the resulting model and probabilities in these two options are identical. Readers not interested in the derivation and discussion of these options can skip to the last paragraph of this section.

In the first option we use positive-operator valued measures (POVMs), a well known measurement framework within quantum theory, in which non-orthogonal outcome vectors can be used. These POVMs allow us to easily build our smaller model with our newly defined belief space. For an introduction to these POVMs, as well as the mathematical details and recipe on how to construct them, we refer to Yearsley (2016, Section 4). The following derivations rely on the derived probabilities given in Yearsley (2016, Equation 56).

In short, when using the POVM framework, the measurement outcome is still represented by an outcome vector and its associated projector. If an outcome is observed, the state vector is still projected onto the relevant subspace; however, the probability of obtaining this outcome is calculated slightly differently. Assume that the player is represented by a state vector $|S\rangle$, the probability of the player thinking that $i$ opponents have cooperated is now:

$$
P^{\prime}\left(B_{i}\right)=\frac{\left\langle B_{i} \mid S\right\rangle^{2}}{\sum_{j=0}^{9}\left\langle B_{j} \mid S\right\rangle^{2}} .
$$

This form deviates from the probabilities derived in section 3.2 only in the factor $\sum_{j=0}^{9}\left\langle B_{j} \mid S\right\rangle^{2}$. This extra factor finds root in the fact that the projectors $P_{j}$ forming a POVM need to adhere to completeness:

$$
\sum_{j=0}^{9} P_{j}=\mathbb{I}
$$


with II the identity matrix. Equation 16 shows that the projectors onto our belief vectors can never sum to the identity matrix, as the off-diagonal elements can never sum to zero. To make sure that the relevant projectors still form a POVM, a new projector (and outcome) is added to the formalism. This projector is associated with the outcome 'measurement failed'. When this outcome is obtained the measurement is redone, ensuring completeness. For details, see again Yearsley (2016).

The second option dismisses the idea of an extra 'measurement fails' outcome and allows the set of projectors $\left|B_{i}\right\rangle\left\langle B_{i}\right|$ to violate the completeness criteria. This violation makes the probabilities of our possible belief outcomes not sum to one:

$$
\sum_{j=0}^{9} P\left(B_{i}\right)=\left\langle B_{i} \mid S\right\rangle^{2} \neq 1 .
$$

From a modeling point of view, this requires the introduction of a scaling factor. This makes sure that the total sum of probabilities does sum to one, after the standard quantum measurement (calculating probabilities and projecting the state vector) is done. This scaling factor is defined as:

$$
C=\sum_{j=0}^{9}\left\langle B_{j} \mid S\right\rangle^{2},
$$

making the probability of eliciting belief $i$, given the state vector $|S\rangle$ :

$$
P^{\prime}\left(B_{i}\right)=\left\langle B_{i} \mid S\right\rangle^{2} / C .
$$

It is vital to note that the end result of both approaches is identical. We have ten outcome vectors, representing the ten possible beliefs, in a two dimensional Hilbert Space $\mathcal{H}_{B}$. The probability of eliciting the belief that $i$ opponents have 
cooperated, given the state vector $|S\rangle$ is:

$$
P^{\prime}\left(B_{i}\right)=\frac{\left\langle B_{i} \mid S\right\rangle^{2}}{\sum_{j=0}^{9}\left\langle B_{j} \mid S\right\rangle^{2}} .
$$

If the result is $i$, the state vector gets projected onto $\left|B_{i}\right\rangle$. The difference between the two options lies in the difference between an approach where we remain firmly within the quantum theoretic setting at the cost of adding an ad hoc new outcome (actually not present in the experimental setting) and an approach slightly departing from the quantum sphere by redefining the probabilities with an ad hoc scaling factor, but having a clear interpretation of all the elements of its machinery regarding the experiment. The choice between the options has no effect on the rest of the paper.

\subsection{The $Q P \mathcal{E} B$ model}

With our belief measurement now adequately defined in the two dimensional $\mathcal{H}_{B}$, we can define $\mathcal{H}_{Q P \& B}$. We still assume the second move action and the belief elicitation to be complementary, representing them by different bases in the redefined 2 dimensional Hilbert Space $\mathcal{H}_{I I, B}$. Additionally, we define the angle between $\left|C_{I I}\right\rangle$ and $\left|B_{9}\right\rangle$ as $\beta_{S M}$ (see Figure 4) and derive estimated probabilities for a player replying that he thinks $i$ opponents cooperate, after the player has cooperated or defected on his second move. As such, this models the consensus effect. We expect $\beta_{S M}$ to be close to 0 , as the consensus effect tells us that people who cooperate are more likely to assume that opponents cooperate as well.

Now we can derive the estimated probabilities for the beliefs of a player 


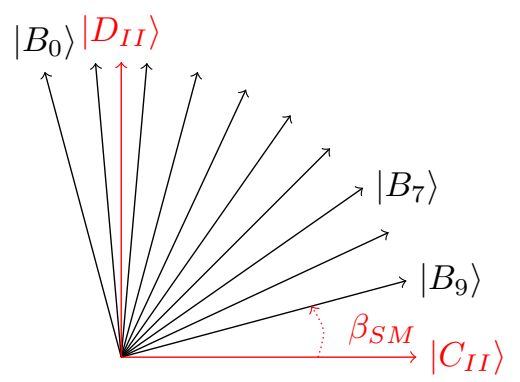

Figure 4: The redefined $\mathcal{H}_{I I, B}$ with both an action-basis and the new belief-basis.

defecting on his second move (making the state vector $|S\rangle=\left|D_{I I}\right\rangle$ ):

$$
\begin{aligned}
P\left(B_{i} \mid D\right) & =\left\langle B_{i} \mid D_{I I}\right\rangle^{2} / \sum_{j=0}^{9}\left\langle B_{j} \mid D\right\rangle^{2} \\
& =\cos ^{2}\left(\beta_{S M}+\frac{i}{9} \frac{\pi}{2}\right) / \sum_{j=0}^{9}\left\langle B_{j} \mid D\right\rangle^{2}
\end{aligned}
$$

and for the beliefs of a player cooperating on his second move (making the state vector $\left.|S\rangle=\left|C_{I I}\right\rangle\right)$ :

$$
\begin{aligned}
P\left(B_{i} \mid C\right) & =\left\langle B_{i} \mid C_{I I}\right\rangle^{2} / \sum_{j=0}^{9}\left\langle B_{j} \mid C\right\rangle^{2} \\
& =\sin ^{2}\left(\beta_{S M}+\frac{i}{9} \frac{\pi}{2}\right) / \sum_{j=0}^{9}\left\langle B_{j} \mid C\right\rangle^{2},
\end{aligned}
$$

with $i \in\{0, \ldots, 9\}$.

Similarly, we define $\mathcal{H}_{I, B}$ as 2-dimensional with both a first move action basis and a belief basis, with $\beta_{F M}$ the angle between $\left|C_{S M}\right\rangle$ and $\left|B_{9}\right\rangle$. Once again, we assume $\beta_{F M}$ close to zero, as players who explicitly think that their opponent will defect are assumed to be more likely to defect as well. We can now derive the estimated probabilities of a player cooperating or defecting on his first moves, after replying that he thinks $i$ opponents cooperated on their second move, which 
made the state vector $|S\rangle=\left|B_{i}\right\rangle$. Note that this first move measurement uses the simple derived probabilities as defined in Section 3.2, as this measurement has both outcome vectors orthogonal.

$$
\begin{aligned}
P\left(D \mid B_{i}\right) & =\left\langle D_{I} \mid B_{i}\right\rangle^{2} \\
& =\cos ^{2}\left(\beta_{F M}+\frac{i}{9} \frac{\pi}{2}\right) .
\end{aligned}
$$

The first and second moves are still considered to be compatible, allowing for a tensoring of their respective Hilbert spaces to represent their correlation. The projectors and probabilities associated with these measurements are identical to the ones defined in the quantum-like model from section 3.2. This gives us a final model $\mathcal{H}_{Q P \& B}=\mathcal{H}_{I, B} \otimes \mathcal{H}_{I I, B}$. In $\mathcal{H}_{Q P \& B}$, the belief that all opponents cooperate is represented by a plane $\boldsymbol{B}_{9}$. The angle between $\boldsymbol{B}_{\mathbf{9}}$ and the plane representing second move cooperation is $\beta_{S M}$. The angle between $\boldsymbol{B}_{\mathbf{9}}$ and the plane representing first move cooperation is $\beta_{F M}$. This also defines the plane $\boldsymbol{B}_{\mathbf{0}}$, which is orthogonal to $\boldsymbol{B}_{\mathbf{9}}$, naturally representing the belief of all opponents defecting and the planes $\boldsymbol{B}_{\boldsymbol{i}}$ between $\boldsymbol{B}_{\mathbf{9}}$ and $\boldsymbol{B}_{\mathbf{0}}$. This incorporates the representation of all three measurements and their relationships (compatible or complementary) into one 4-dimensional Hilbert space, with clear estimated probabilities resulting from this representation.

\subsection{Fitting the data}

We fit the experimental data of the three measurements to our model. Note that the proportions of the second move actions are already incorporated in the starting state vector (equation 15). Since we have derived concrete dependencies of the beliefs on the second moves, and of the first moves on the beliefs, we can formally 
fit the experimental data of the Elicit_Beliefs group to our model. To do so, we shall estimate an optimal value of $\beta$ for the beliefs on the second moves, as well as for the first moves on the beliefs. This can be achieved by minimizing the distance between the counts observed in our data set and the expected frequencies based on the equations derived above. The chi-squared test is typically used to check whether or not an observed set of proportions sufficiently matches the expected set, so we will focus on minimizing this statistic.

Let us first focus on the two contingency tables representing the dependencies of the beliefs on the second moves (see Tables 4 and 5). When a specific value of $\beta_{S M}$ is provided, we can estimate the expected probabilities $P\left(B_{i} \mid D\right)$ and $P\left(B_{i} \mid C\right)$ based on equations (23) and (25), respectively, and subsequently evaluate a chisquared statistic for each of the two tables. In order to estimate an appropriate $\beta_{S M}$, we optimize an algorithm in which the sum of the two chi-squared statistics (one for the SM defectors and one for SM cooperators) is minimized over a range of possible values for $\beta_{S M}$ (ranging from $-\pi / 2$ to $\pi / 2$ ). The value of $\beta_{S M}$ for which this sum reaches its lowest point equals -0.2048 , corresponding to chi-squared statistics of 14.13 and 14.24 for the two contingency tables (one concerning the second move cooperators and one concerning the second move defectors), respectively. As expected, our estimated $\beta_{S M}$ is indeed near 0 .

Under normal circumstances, these chi-squared statistics can be translated into p-values, by relying on their asymptotic approximation of a chi-squared distribution with $I-1$ degrees of freedom (with $I=10$ the number of possible beliefs). For our data set, however, this asymptotic procedure can be problematic because several of the expected frequencies fall below five. This induces concern about the accuracy of any p-value obtained through asymptotic approximation; 


\begin{tabular}{c|cccccccccc}
$\mathrm{i}$ & 0 & 1 & 2 & 3 & 4 & 5 & 6 & 7 & 8 & 9 \\
\hline Observed counts & 1 & 0 & 0 & 0 & 3 & 6 & 9 & 6 & 4 & 3 \\
Observed proportions & 0.031 & 0.000 & 0.000 & 0.000 & 0.094 & 0.188 & 0.281 & 0.188 & 0.125 & 0.094 \\
Expected proportions & 0.011 & 0.000 & 0.005 & 0.025 & 0.058 & 0.099 & 0.144 & 0.187 & 0.223 & 0.248
\end{tabular}

Table 4: The observed counts, as well as the observed and expected proportions of the beliefs of second move cooperators

\begin{tabular}{c|cccccccccc}
$\mathrm{i}$ & 0 & 1 & 2 & 3 & 4 & 5 & 6 & 7 & 8 & 9 \\
\hline Observed counts & 4 & 2 & 5 & 5 & 9 & 3 & 0 & 0 & 0 & 0 \\
Observed proportions & 0.143 & 0.071 & 0.179 & 0.179 & 0.321 & 0.107 & 0.000 & 0.000 & 0.000 & 0.000 \\
Expected proportions & 0.156 & 0.163 & 0.160 & 0.147 & 0.127 & 0.101 & 0.072 & 0.045 & 0.022 & 0.007
\end{tabular}

Table 5: The observed counts, as well as the observed and expected proportions of the beliefs of second move defectors

therefore, we resort to a more accurate estimation via Monte Carlo simulation. This technique simulates the sampling distribution of the test statistic (in this case, chi-squared) using Monte Carlo methods. We generate random contingency tables with the same marginal distribution as our data (i.e. the same sample size), and calculate their chi-squared statistic. Subsequently, it is determined how many of these random samples display a test-statistic which is larger than the one that was originally obtained. The resulting proportion of more extreme chi-squared statistics represents our new and more accurate p-value. Note that what can be calculated for one chi-squared statistic can also be achieved for a sum of chi-squared statistics: we can simulate a p-value corresponding to the proportion of summed test-statistics, which are larger than the original sum $(14.13+14.24=28.37)$. For our analyses, we chose to rely on $10^{4}$ simulated samples.

According to the reasoning in the previous paragraph, these two test-statistics allow us to calculate a p-value through Monte Carlo simulation: we obtain one of 0.071 for both tables combined. Their observed counts, alongside the observed 


\begin{tabular}{c|ccccc}
$\mathrm{i}$ & Observed counts & Totals & Observed proportions & Expected proportions & $\chi^{2}$ \\
\hline 0 & 5 & 5 & 1.000 & 0.997 & 0.016 \\
1 & 2 & 2 & 1.000 & 0.947 & 0.111 \\
2 & 5 & 5 & 1.000 & 0.844 & 0.925 \\
3 & 5 & 5 & 1.000 & 0.699 & 2.153 \\
4 & 6 & 12 & 0.500 & 0.530 & 0.044 \\
5 & 3 & 9 & 0.333 & 0.358 & 0.023 \\
6 & 1 & 9 & 0.111 & 0.202 & 0.464 \\
7 & 0 & 6 & 0.000 & 0.083 & 0.542 \\
8 & 0 & 4 & 0.000 & 0.014 & 0.056 \\
9 & 0 & 3 & 0.000 & 0.003 & 0.010
\end{tabular}

Table 6: The observed number of cooperators, total number of participants, and observed as well as expected proportions of and expected proportions of first move cooperators. Note that the observed number of defectors (as well the the respective observed and expected frequencies) are not mentioned in this table since this information is redundant (the observed counts of cooperators and defectors sum to the totals and the observed/expected proportions of both defectors and cooperators sum to one).

and expected frequencies, can be found in Tables 4 and 5. The p-value testing the null hypothesis of no significant difference between our observed and expected proportions on the $\alpha=0.05$ level indicates an acceptable fit. As this p-value is estimated using simulation, the degrees of freedom are not taken into account, unlike a traditional (asymptotic) p-value where the chi-square distribution is used. As such, this p-value does not take into account that 20 proportions are estimated using only one free parameter, making our estimated p-value even more favorable to accepting the null hypothesis than the value suggests at first sight. See Tables 4 and 5 .

When we aim to establish an optimal value of $\beta_{F M}$ for modeling the first move actions, we see that we have to deal with ten different contingency tables: one for each belief in the number of cooperators $(i=0, \ldots, 9)$. Since the observed and expected probabilities in each of these contingency tables sum to one, we only 


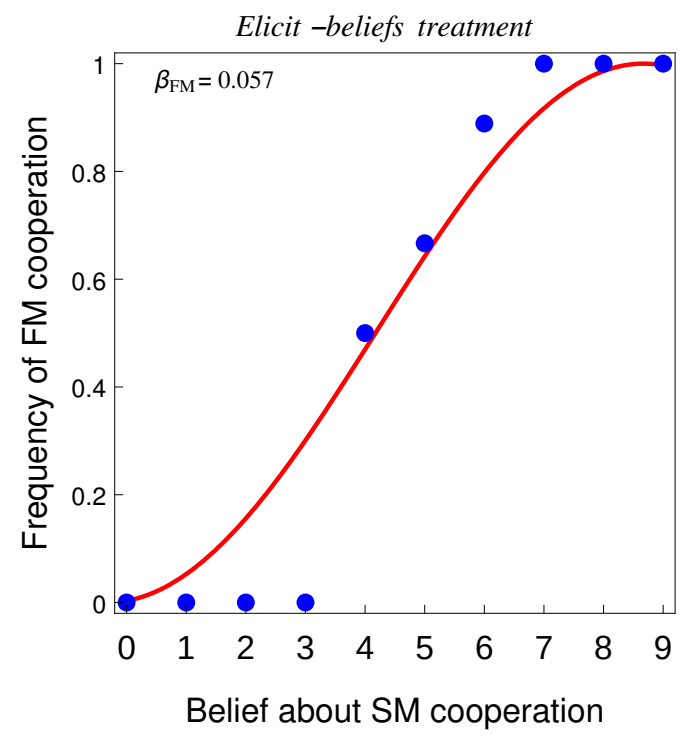

Figure 5: Observed frequency of FM cooperation versus elicited beliefs on SM cooperation (blue dots) and fitted model (equation 27, red line).

need to focus on the data counts and proportions for the cooperators $P\left(C \mid B_{i}\right)$. Similar to the beliefs of the second moves, we establish an optimal value of $\beta_{F M}$ for the first move cooperators by minimizing the sum of the ten chi-squared statistics using equation (27). The optimal value of $\beta_{F M}$ is 0.057 which is close to 0 , as expected. Figure 5 plots the analytical prediction of the POVM model (equation 27) for the relationship between first move cooperation rates and stated beliefs about second mover cooperation with $\beta_{\mathrm{FM}}=0.057$, and compares it to the experimental observations. ${ }^{10}$ The chi-squared statistics and expected proportions are displayed in Table 6; and the corresponding simulated p-value equals 0.715 indicating a very good fit.

\footnotetext{
${ }^{10}$ Blanco et al. (2014, Figure 3) explain the observed relationship between both experimental variables with a probit regression, obtaining a similar dependency. Nevertheless, our analytical curve has a deeper meaning because the functional form (equation 27) is a direct consequence of the geometrical structure of the POVM model.
} 


\section{Discussion}

Our decision to abandon the restriction that outcome vectors coming from one measurement are orthogonal to each other has consequences. The most important one is the loss of the repeatability of outcomes. ${ }^{11}$ Repeatability entails that when a measurement is performed twice (without any manipulation or evolution of the system between two measurements), the same outcome is observed twice. This is assured in a standard quantum-like model, as the projection of a state vector onto an orthogonal subspace gives the null vector. Repeatability seems a very logical and sensible restriction but has been called into question, specifically when applied in quantum cognition. See for example Khrennikov et al. (2014) for a thorough discussion of this problem and Aliakbarzadeh and Kitto (2016) about the use of POVMs, which lack repeatability, in Social Sciences.

In our context, the loss of repeatability in the measurement of beliefs means that when a player replies that, e.g., 'six' opponents cooperate, he might reply 'seven' when the question would be posed again. To justify this, we consider the measurements to be unsharp. Unsharp measurements are measurements such that the outcome represents a bigger subset of a (possible non-discrete) set of outcomes. This is applicable in cases where a subject is asked to form a precise opinion or belief but he is actually forming a broader opinion or belief. For our example of interest, the subjects may think 'most of them' but have to give a discrete number as an answer. We assume that when a player replies that, e.g., six out of nine opponents cooperate, this indicates the player believing 'somewhere around six out of nine opponents cooperate'. This implies that he

\footnotetext{
${ }^{11}$ Also called first kindness in Danilov and Lambert-Mogiliansky (2008).
} 
would not necessarily disagree with the opinion that seven out of nine opponents have cooperated. This structure can be viewed in the form of the belief vectors $\left|B_{i}\right\rangle$. The state vector collapsing on $\left|B_{6}\right\rangle$ does not preclude the outcome associated with $\left|B_{7}\right\rangle$, as they are close to each other, with the angle between them equaling $\pi / 18$. The closer two vectors are to being orthogonal, the more the outcomes they represent do preclude each other. The vectors $\left|B_{0}\right\rangle$ and $\left|B_{9}\right\rangle$ are the limit case: being orthogonal makes the events associated with them (the opponent cooperating and defecting for sure) completely preclude each other.

The use of these non-orthogonal outcome vectors also opens up new research possibilities within quantum cognition. Inflated dimensionality is a common obstacle in elegant model building. Once multiple (compatible) measurements with more than two possible outcomes are taken into account, any standard quantum model would require high dimensionality. Next to the ease of reducing dimensionality, some extra structure can be incorporated in the model. As can be seen in our case, implicit relationships between different outcomes can be represented. Our model can have the $B_{1}$ outcome be 'closer' to the $B_{2}$ outcome than it is to the $B_{8}$ outcome. In a standard quantum model, all outcome vectors are orthogonal, so all outcomes play a similar role towards each other. This works for all discrete examples in physics, but in decision-making there are numerous examples of ordinal scales where a kind of structure is implied between the different outcomes. While this might have seemed problematic at first, there are other examples in which these techniques are successfully used, again, see Aliakbarzadeh and Kitto (2016) and Yearsley (2016). As there is, to our knowledge, no known way of simply incorporating ordinal scales into the quantum framework (preserving some sense of the notion of an order), 
constructing bases similar to the one in this paper seems to be an interesting road for future research. One obvious candidate for this treatment would be the quantum-like modeling of Likert scales. They allow for (mostly) 5 or 7 different ordered outcomes and are ordinal scales widely used within cognition. Some first steps for Likert scales of this form are presented in Yearsley (2016).

\section{Conclusion}

In this paper we constructed a quantum-like model for preferences and beliefs in a social dilemma game. By taking a new look at the experimental data set collected by Blanco et al. (2014) for a sequential prisoner's dilemma, we identified and discussed three distinct effects. These effects are all explained as a specific type of relationship between the measurements performed in the experiment. First, there is a direct positive correlation between the player's first and second move. As it is shown in Blanco et al. (2014), however, this does not provide a complete picture of the subject's behavior because this correlation is also driven by an indirect belief-based channel. This interaction is made up of the two other effects: the influence of the second move on the beliefs of the player and the influence of the beliefs of the player on his first move. The former effect is the socalled consensus effect and we attributed the latter effect to the player becoming more reasoned about his preferences.

The nature of these last two effects both point towards a quantum-like model. The non-classical structure of the consensus effect is already discussed in Busemeyer and Pothos (2012) where it is viewed as a form of social projection. Busemeyer and Pothos (2012) represented the construction of a player's belief as a rotation of the state vector in a Hilbert Space. In our case, the belief construction and the second move action are non-commuting (and thus incompatible) in nature. 
The effect of the player becoming more reasoned can be seen as a violation of the sure thing principle. The act of belief elicitation significantly changes the cooperation rate of the first move action, regardless of the beliefs elicitation outcome. This also suggests viewing the belief elicitation and the first move action as incompatible. We combined all these observations and constructed a Hilbert Space in which the action measurements on the one hand and the belief measurements on the other hand were viewed as incompatible measurements by defining a different basis for each.

Following the more traditional recipe, we obtained a model within a 100dimensional Hilbert Space which was greatly overparametrized from a statistical point of view. As a solution to this problem, we proposed to redefine the belief basis as two-dimensional and considered two options. The first option constructed a POVM, which framed our model neatly into conventional quantum theory, at the cost of defining an ancillary outcome. The second option dismissed this new outcome, staying closer to the actual experiment, at the cost of leaving the standard quantum-like framework. Both options result in identical models and diminish the problematic dimensionality. This model incorporates the three effects observed in the experiment, and yields elegant dependencies between actions and beliefs with successful statistical fit.

As not all vectors associated with outcomes of the belief measurement were orthogonal, we lose repeatability of outcomes: obtaining an outcome does not exclude obtaining a different outcome when the same measurement is performed again immediately. We defined unsharp measurements of beliefs where forcing the player to pick one outcome does not mean he disagrees with some other possible outcome, thus relaxing the constraint of repeatability. For more on 
the need (or lack thereof) of repeatability in psychological measurements, see Khrennikov et al. (2014).

\section{Acknowledgements}

We are grateful to the authors of the original experiment (Blanco et al., 2014) and especially to Hans-Theo Normann for providing access to their experimental data set. We would also like to thank two anonymous referees, the Special Issue Guest Editor, Sandro Sozzo, and the Associate Editor, Ehtibar Dzhafarov, for helpful comments; as well as James Yearsley and Ariane Lambert-Mogiliansky for fruitful discussions.

This work has been partly supported by COST Action IC1205 on Computational Social Choice. ABD was supported by Air Force Office of Scientific Research (AFOSR), Air Force Material Command, USAF, grant FA 8655-13-1-3044.

\section{References}

Aerts, D., 2009. Quantum structure in cognition. Journal of Mathematical Psychology 53 (5), 314-348.

Aerts, D., Gabora, L., 2005. A theory of concepts and their combinations II: A Hilbert space representation. Kybernetes 34 (1/2), 192-221.

Aliakbarzadeh, M., Kitto, K., 2016. Applying povm to model non-orthogonality in quantum cognition. In: Atmanspacher, H., Filk, T., Pothos, E. (Eds.), Quantum Interaction. Vol. 9535 of Lecture Notes in Computer Science. Springer International Publishing, pp. 284-293. 
Atmanspacher, H., Filk, T., Römer, H., 2004. Quantum Zeno features of bistable perception. Biological Cybernetics 90 (1), 33-40.

Barque-Duran, A., Pothos, E. M., Yearsley, J., Hampton, J., Busemeyer, J. R., Trueblood, J. S., 2016. Similarity Judgments: From Classical to Complex Vector Psychological Spaces. In the series: Advanced Series on Mathematical Psychology by E. Dzhafarov, R. Zhang, S. Joardan, and V. Cervantes (Eds.) Contextuality from Quantum Physics to Psychology. World Scientific.

Blanco, M., Engelmann, D., Koch, A., Normann, H.-T., 2014. Preferences and beliefs in a sequential social dilemma. Games and Economic Behavior 87, 122135.

Blutner, R. K., 2008. Concepts and Bounded Rationality: An Application of Niestegge's Approach to Conditional Quantum Probabilities. In L. Accardi, et al. (eds.), Foundations of Probability and Physics 5, Vol. 1101, pp. 302-10. American Institute of Physics Conference Proceedings.

Bohr, N., 1950. On the notions of causality and complementarity. Science 111(2973)), 51-54.

Bordley, R., 1998. Quantum mechanical and human violations of compound probability principles: toward a generalized Heisenberg uncertainty principle. Operations Research 46, 923-926.

Brandenburger, A., 2005. The relationship between quantum and classical correlation in games. Games and Economic Behavior 69(1), 175-183.

Brunner, N., Linden, N., 2013. Connection between Bell nonlocality and Bayesian game theory. Nature Communications 4(2057), 1-6. 
Bruza, P. D., 2010. Quantum memory. Australasian Science 31 (1), 34-35.

Bruza, P. D., Kitto, K., Nelson, D., McEvoy, C. L., 2009. Is there something quantum-like about the human mental lexicon? Journal of Mathematical Psychology 53, 362-377.

Busemeyer, J., Bruza, P., 2012. Quantum Models of Cognition and Decision. Cambridge University Press, Cambridge.

Busemeyer, J., Pothos, E., 2012. Social projection and a quantum approach for behavior in Prisoner's Dilemma. Psychological Inquiry 23 (1), 28-34.

Busemeyer, J., Pothos, E., Franco, R., Trueblood, J., 2011. A quantum theoretical explanation for probability judgment errors. Physchological Review 118(2), 193-218.

Busemeyer, J., Wang, Z., Townsend, J., 2006. Quantum dynamics of human decision-making. Journal of Mathematical Psychology 50, 220-241.

Danilov, V. I., Lambert-Mogiliansky, A., 2008. Measurable systems and behavioral sciences. Mathematical Social Sciences 55 (3), 315-340.

Denolf, J., Lambert-Mogiliansky, A., 2016. Bohr complementarity in memory retrieval. Vol. 73 .

Deutsch, D., 1999. Quantum theory of probability and decisions. Proceedings of the Royal Society A 455, 3129-3137.

Hameroff, S. R., 2007. The brain is both neurocomputer and quantum computer. Cognitive Science 31 (6), 1035-1045. 
Haven, E., Khrennikov, A., 2013. Quantum social science. Cambridge University Press.

Hughes, R. I. G., 1989. The structure and interpretation of quantum mechanics. Harvard University Press.

Isham, C. J., 1989. Lectures on quantum theory. Singapore: World Scientific.

Khrennikov, A., 2010. Ubiquitous Quantum Structure: From Psychology to Finance. Springer, Berlin.

Khrennikov, A., Basieva, I., Dzhafarov, E. N., Busemeyer, J. R., 2014. Quantum models for psychological measurements: an unsolved problem. PloS ONE 9 (10), e110909.

La Mura, P., 2005. Correlated equilibria of classical strategic games with quantum signals. International Journal of Quantum Information 3 (01), 183-188.

Lambert-Mogiliansky, A., Martínez-Martínez, I., 2015. Games with Type Indeterminate players: a Hilbert space approach to uncertainty and strategic manipulation of preferences. In: Atmanspacher, H., Bergomi, C., Filk, T., Kitto, K. (eds.) QI 2014. Lecture Notes in Computer Science 8951. Springer International Publishing Switzerland.

Lambert-Mogiliansky, A., Zamir, S., Zwirn, H., 2009. Type indeterminacy: a model for the KT(Kahneman-Tversky)-man. Journal of Mathematical Psychology 53, 349-361.

Lichtenstein, S., Slovic, P., 2006. The construction of preference. Cambridge University Press, New York. 
Litt, A., Eliasmith, C., Kroon, F. W., Weinstein, S., Thagard, P., 2006. Is the brain a quantum computer? Cognitive Science 30 (3), 593-603.

Martínez-Martínez, I., Denolf, J., Barque-Duran, A., 2015. Do preferences and beliefs in dilemma games exhibit complementarity? In: International Symposium on Quantum Interaction. Springer, pp. 142-153.

Martínez-Martínez, I., Sánchez-Burillo, E., 2016. Quantum stochastic walks on networks for decision-making. Scientific Reports 6 (23812).

Pothos, E., Busemeyer, J., 2009. A quantum probability explanation for violations of 'rational' decision theory. Proceedings of the Royal Society of London B: Biological Sciences, rspb-2009.

Pothos, E., Perry, G., Corr, P., Matthew, M., Busemeyer, J., 2011. Understanding cooperation in the Prisoners Dilemma game. Personality and Individual Differences 51(3), 210-215.

Pothos, E., Yearsley, J., Barque-Duran, A., Hampton, J., Busemeyer, J., Trueblood, J., 2015. Progress and current challenges with the Quantum Similarity Model. Frontiers in Psychology, 6:205.

Pothos, E. M., Busemeyer, J. R., 2013. Can quantum probability provide a new direction for cognitive modeling? Behavioral and Brain Sciences 36, 255-327.

Trueblood, J., Busemeyer, J., 2011. A quantum probability account of order effects in inference. Cognitive Science 35, 1518-1552.

Wang, Z., Solloway, T., Shiffrin, R., Busemeyer, J., 2014. Context effects 
produced by question orders reveal quantum nature of human judgments? Proceedings of the National Academy of Sciences USA 111(26), 9431-9436.

White, L., Barque-Duran, A., Pothos, E., 2015. An investigation of a quantum probability model for the constructive effect of affective evaluation. Philosophical Transactions of the Royal Society A.

White, L., Pothos, E., Busemeyer, J., 2014. Sometimes it does hurt to ask: the constructive role of articulating impressions. Cognition 133, 48-64.

Yearsley, J., Pothos, E., 2014. Challenging the classical notion of time in cognition: a quantum perspective. Proceedings of the Royal Society B 281), 20133056.

Yearsley, J. M., 2016. Advanced tools and concepts for quantum cognition: A tutorial. Journal of Mathematical Psychology. Special Issue on Quantum Probability (in press).

Yearsley, J. M., Pothos, E. M., Hampton, J., Barque-Duran, A., 2014. Towards a quantum probability theory of similarity judgments. Proceedings of the Quantum Interaction Conference.

Yukalov, V. I., Sornette, D., 2011. Decision theory with prospect interference and entanglement. Theory and Decision 70 (3), 283-328. 